\title{
An Analytical Study of Variable Preamble Length-Based Broadcasting Scheme for WSNs
}

\author{
Arun Kumar ${ }^{1}$, Kai-Juan Wong ${ }^{2}$ \\ ${ }^{1}$ School of Computer Engineering, Nanyang Technological University, Singapore \\ ${ }^{2}$ Singapore Institute of Technology, Singapore \\ Email: arun0020@e.ntu.edu.sg, steven.wong@singaporetech.edu.sg
}

Received May 2013

\begin{abstract}
In this paper, we present a detailed analytical study of previously proposed variable preamble length-based broadcasting scheme for wireless sensor networks (WSNs). Analytical results show that in dense wireless sensor networks, broadcasting a packet with a small size preamble can significantly improve the energy conservation on already limited battery powered sensor nodes. For fairer comparison with variable preamble length-based broadcasting scheme, a comprehensive analytical study for an existing probability-based broadcasting scheme is also presented. Analytically calculated results show that variable preamble length-based broadcasting scheme is more energy-efficient than the probabilitybased broadcasting scheme.
\end{abstract}

Keywords: Broadcast; Flooding; Analytical Model; Wireless Sensor Network

\section{Introduction}

A large-scale wireless sensor network (WSN) can consist of thousands of sensor nodes and are helpful in many situations [1], such as disaster relief missions and battlefield communication facilities, infrastructure protection and scientific exploration [1-3]. Wireless data transmission consumes more energy than data processing in a sensor node. The situation becomes worse in dense wireless networks where broadcasting of data is necessary. In such cases, transmission of a single bit may consume the same amount of energy as that needed for processing thousand operations in a typical sensor node.

Wireless sensor networks have broadcast as one of its most fundamental service. Broadcast provides maximum message propagation across the whole network and serves high-level operations, making it critical to the overall network design. Although broadcasting in wireless sensor networks have many advantages, it can cause serious problems like "broadcast storm problem" [4], which could cause a lot of contention, redundant retransmission, collision and most importantly, waste immense amount of energy. A possible solution is that the sensor nodes alternate between active and dormant states, which help them to conserve energy and extend the network lifetime. Simple broadcasting in this environment, where a node goes to active and dormant state periodically in asynchronous manner can be very challenging if there are not enough nodes in the active state when a source wants to send data.

As wireless communication is the major cause of energy consumption on sensor nodes, efforts have focused on how to conserve energy especially at the medium access control (MAC) layer. While a long preamble is used before the data packet in BMAC [5], we believe that a small preamble is enough to achieve sufficient reachability and throughput in dense wireless area networks. On the other hand, it can dramatically save the energy required to broadcast a packet.

In this paper, we present an analytical study for variable preamble length-based broadcasting scheme [6]. An Analytical study for an existing probability-based broadcasting scheme is also presented and the scheme is implemented to compare with the variable preamble lengthbased scheme.

The rest of the paper is organized as follows. Section 2 introduces the motivation behind variable preamble lengthbased broadcasting scheme. In Section 3, an analytical study for variable preamble length-based and probability-based broadcasting schemes is given. The parameters used during the simulations and results are given in Section 4 . Section 5 concludes the paper and gives some suggestions for future work.

\section{Motivation and Variable Preamble Length-Based Broadcasting}

Wireless sensor networks have an important property that 
many sensor nodes alternate between active and dormant states, helping them to conserve energy and extend network lifetime. In dense area networks, there is a greater chance of a node being in an active state to listen to the channel at a particular time, when compared to a sparse area network. Simple flooding in dense area networks, where more nodes are active at a time near the relay node will only cause energy consumption rather than covering additional areas in the network, resulting in unnecessary retransmission of packets and collisions.

Energy efficient MAC protocols are used to conserve energy of sensor nodes in wireless sensor networks. BMAC [5] is an energy-saving, distributed, asynchronous and random-access MAC protocol best suited for wireless sensor networks. BMAC employs the idea of "preamble listening" to conserve energy, whereby nodes would duty-cycle the radio receivers by periodically sampling the channel for activity. In order to transmit a packet, a node would transmit a long preamble to inform its neighbors of impending data. This allows B-MAC to support asynchronous and random-access transmission of data packets. However, a long preamble contains no useful data and in dense wireless networks it is the major cause of energy wastage. Preamble length $T_{\text {Preamble }}$ in BMAC must be such that

$$
T_{\text {Preamble }}>T_{\text {Interval }}
$$

where $T_{\text {Preamble }}$ is the duration of preamble length and $T_{\text {Interval }}$ is the wakeup interval of the nodes.

Variable preamble length-based broadcast uses a small preamble before the data packet in dense wireless sensor networks [6]. Figure 1 shows the difference between (a) broadcast using the long preamble before the data packet and (b) broadcast using variable preamble length in asynchronous duty cycled wireless sensor network. The small preamble can clearly affect the overall performance of the broadcasting scheme in dense networks. In case of small preamble being sent before the data packet, this preamble will only be listened by a subset of nodes present in the radio range of a relay node or sender node. The subset of sensor nodes that receives the data packet then rebroadcasts the packet using the same preamble length. This practice of sending a small preamble before the data packet reduces the number of rebroadcasts as well as the overall energy consumption in the network, as only a subset of nodes will listen to the preamble and receive the data packet. Sensor nodes will rebroadcast the packet only after a random delay.

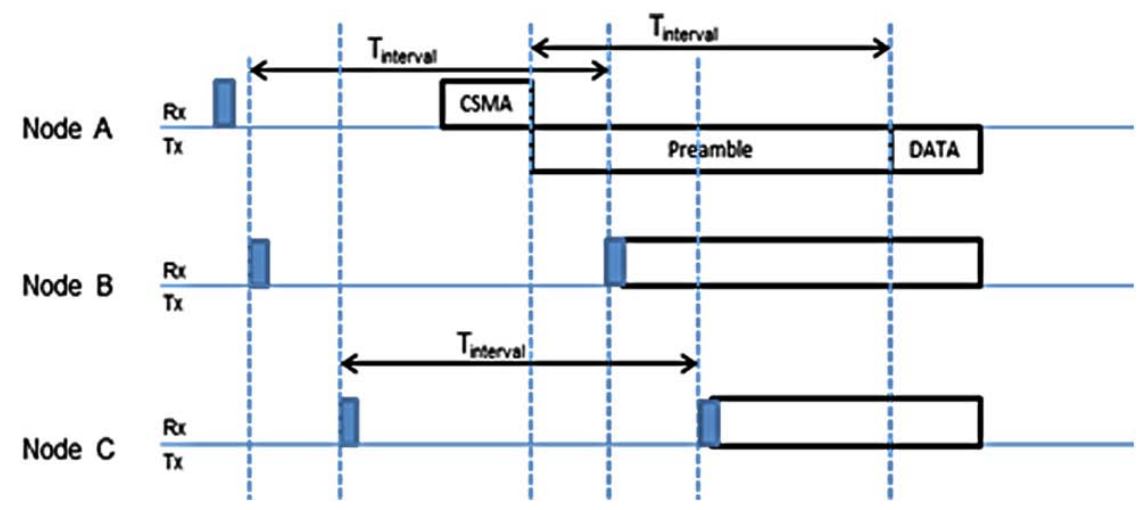

(a)

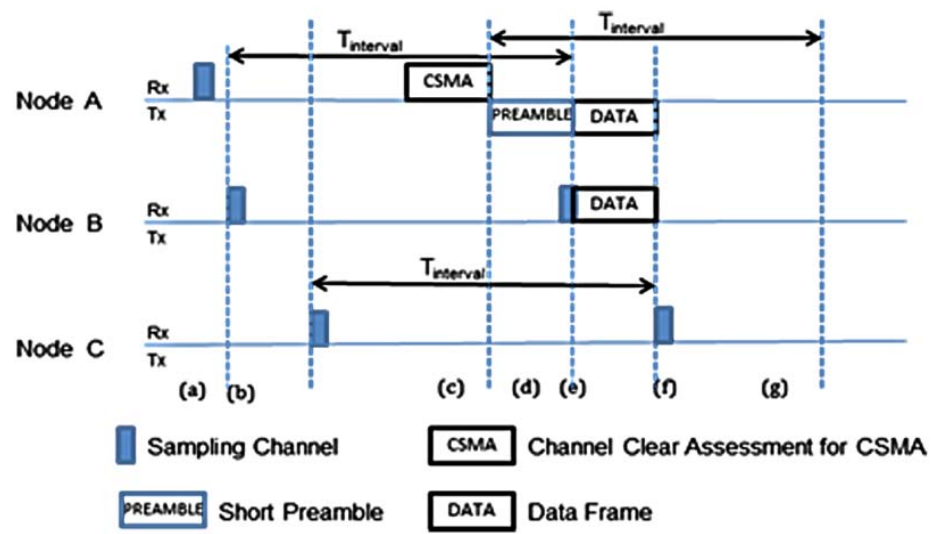

(b)

Figure 1. Shows the difference between (a) Broadcast using the long preamble before the data packet and (b) Broadcast using variable preamble length in asynchronous duty cycled wireless sensor networks. 
In dense area networks, even small preambles used in variable preamble length-based broadcast [6] can give sufficient data dissemination and dramatically save the energy of the node. In sparse area network, a comparatively large preamble will be required due to the fact that there are lesser chances that a node is active in the neighborhood of the relaying node or the sender node. These small preambles will save most of the rebroadcasts and conserve maximum energy in dense area networks. In case of small preamble being used the preamble length $T_{\text {Preamble }}$ must be such that

$$
T_{\text {Preamble }} \leq T_{\text {Interval }}
$$

where $T_{\text {Preamble }}$ is the duration of the preamble and $T_{\text {Interval }}$ is the wakeup interval of the nodes.

\section{Analytical Models}

Most of the energy consumption on a node is because of the node's radio power consumption. Therefore, analytical models focus on the radio power consumption of nodes. Some equations in this section are due to [7].

Table 1 lists the abbreviations and units used in the equations for the calculation of the energy consumption.

Furthermore, to simplify the comparisons, the analytical study is based on the following conditions:

- All equations are normalized to one second.

- All the scenarios have same number of nodes and transmission conditions.
- Both the broadcasting algorithms use preambles before the data packet but the size of the preamble differs substantially. The following equations are used to calculate the duration of $T_{\text {Preamble }}$ and $T_{S_{\text {SPreamble }}}$ for the probability-based and variable preamble length-based broadcasting schemes respectively:

$$
\begin{aligned}
& T_{\text {Preamble }}=T_{\text {Interval }}+T_{\text {Sample }}+T_{\text {Guard }} \\
& T_{S_{\text {_Preamble }}}=T_{\text {Variable }}+T_{\text {Sample }}+T_{\text {Guard }}
\end{aligned}
$$

- To perform channel sampling from an idle state, a node has to turn its radio on and then sample the channel. Thus, the time taken to perform channel sampling is calculated to be:

$$
T_{\text {Sample }}=T_{I R x}+T_{\text {RSSI }}
$$

- A node will turn on its receiver and wait for the Radio Strength Signal Information (RSSI) value to stabilize before transmitting a packet. Then the node will check whether the channel is free for the entire duration of $T_{\text {csma }}$. For simplifying the comparison between the different protocols, the channel is always assumed to be clear, and therefore no back-offs were considered during channel contention. At the MAC layer, for both the broadcasting protocols, the total time, $T_{\text {CSMA }}$, in which the radio receiver is turned on to perform CSMA is calculated as follows:

\begin{tabular}{|c|c|c|}
\hline Abbreviation & Description & Unit \\
\hline$T_{T x}$ & Time taken to transmit a data frame & $\mathrm{Sec}$ \\
\hline$T_{R x}$ & Time taken to receive a data frame & $\mathrm{Sec}$ \\
\hline$T_{S \_ \text {Preamble }}$ & The duration for which the small preamble is sent & $\mathrm{Sec}$ \\
\hline$T_{\text {Variable }}$ & The variable minimum time duration for which the small preamble should be sent & $\mathrm{Sec}$ \\
\hline$T_{\text {Interval }}$ & Time between each sampling of the channel during channel listening & $\mathrm{Sec}$ \\
\hline$T_{\text {Preamble }}$ & The minimum duration for which the preamble should be sent & $\mathrm{Sec}$ \\
\hline$T_{\text {Guard }}$ & A guard time to allow for clock skews between nodes & $\mathrm{Sec}$ \\
\hline$T_{c s m a}$ & Duration for which the channel has to be clear before the node can assume that the channel is free & $\mathrm{Sec}$ \\
\hline$N_{\text {Neigh }}$ & Number of neighbors per node & \\
\hline$N_{P k t s}$ & Number of packets transmitted per second & \\
\hline$T_{T x b}$ & Time taken to transmit a byte & $\mathrm{Sec}$ \\
\hline$T_{R x T x}, T_{I T x}$ & Time taken for the radio to switch from Receive $(R x) /$ Idle to Transmit (Tx) mode & $\mathrm{Sec}$ \\
\hline$T_{I R x}$ & Time taken for the radio to switch from Idle to Receive mode & $\mathrm{Sec}$ \\
\hline$T_{R S S I}$ & Time for $R S S I$ to provide a reading & $\mathrm{Sec}$ \\
\hline$T_{\text {Sample }}$ & Time taken from the idle state to sample the channel for activity & $\mathrm{Sec}$ \\
\hline$P_{R x}$ & Power consumption of the radio in Receive mode & $m W$ \\
\hline$P_{T x}$ & Power consumption of the radio in Transmit mode & $m W$ \\
\hline$P_{\text {Idle }}$ & Power consumption of the radio in Idle Mode & $m W$ \\
\hline$P$ & Predefined probability with which a data packet is broadcasted $[0.2,1]$ & \\
\hline
\end{tabular}

$$
T_{\text {CSMA }}=\left(T_{I R x}+T_{R S S I}+T_{c S m a}\right) * N_{\text {Pkts }}
$$

Table 1. Notations in the analytical model. 
- As in probability-based schemes nodes do not require global topological information of the network to make rebroadcast decisions. Every node is allowed to rebroadcast a packet based on a predetermined forwarding probability $P$. A random number between 0 and 1 is generated and if this random number is below or equal to a predefined probability $P$ then the packet is rebroadcasted.

$$
\text { Random Number } \leq P
$$

- It is assumed that the transmitted signals will always be received correctly. So the channel noise is not modelled in the equations.

- In this analysis, for a fairer comparison of both the broadcasting schemes, the equations and results are based on "average case" scenarios for probability based broadcasting scheme and on the "average or worst case" scenarios for variable preamble length-based broadcasting scheme.

\subsection{Probability-Based Broadcasting Scheme}

The total power consumption of the radio in the case of probability-based broadcasting scheme, $E_{\text {ProbBroadcast }}$ is calculated from the durations for which the radio receiver or transmitter is turned on to perform the following operations: CSMA (4), transmission of the data packet (7), reception of a data packet (8), channel listening (9) and idling (10).

$$
\begin{aligned}
E_{\text {ProbBroadcast }}= & E_{\text {CSMA }}+E_{\text {ProbBroadcast_TX }}+E_{\text {ProbBroadcast_Rx }} \\
& +E_{\text {ProbBroadcast_Listen }}+E_{\text {ProbBroadcast_Idle }}
\end{aligned}
$$

where, $\left(E_{\text {CSMA }}=T_{\text {CSMA }} * P_{R x}\right),\left(E_{\text {ProbBroadcast_Tx }}=T_{\text {ProbBroad- }}\right.$ $\left.{ }_{\text {cast_TX }} * P_{T X}\right),\left(E_{\text {ProbBroadcast_Rx }}=T_{\text {ProbBroadcast_Rx }} * P_{R x}\right),\left(E_{\text {Prob- }}\right.$ Broadcast_Listen $\left.=T_{\text {ProbBroadcast_Listen }}{ }^{*} P_{R x}\right)$ and $\left(E_{\text {ProbBroadcast_Idle }}\right.$ $=T_{\text {ProbBroadcast_Idle }} * P_{\text {Idle }}$ ).

Before transmitting a data packet, a node always sends a long preamble followed by the data packet. Therefore, the time, $T_{\text {ProbBroadcast } T x}$, for which the transmitter will be turned on can be represented by following equation;

$$
T_{\text {ProbBroadcast_Tx }}=\left(T_{R \times T X}+T_{\text {Preamble }}+T_{T x}\right) * N_{\text {Pkts }} * P
$$

To receive a data packet, nodes turn their receiver on if any channel activity is detected during the periodic listening. These nodes will keep their receiver turned on until a packet is received and the total time, $T_{\text {ProbBroad- }}$ cast_Rx, for which the node receiver is turned on for the reception of a data packet is represented by following equation:

$$
\begin{aligned}
& T_{\text {ProbBroadcast } \_R x} \\
= & \left(\left(0.5 * T_{\text {Preamble }}\right)+T_{R x}\right) * N_{\text {Pkts }} * N_{\text {Neigh }} * P
\end{aligned}
$$

For the remainder of the time, when a node is neither receiving nor transmitting data, the node performs peri- odic channel listening. The duration, $T_{\text {ProbBroadcast_Listen, for }}$ which the receiver is turned on for channel listening is calculated as follows:

$$
\begin{aligned}
& T_{\text {ProbBroadcast_Listen }} \\
= & \left\lfloor\left(\left(\left(\left(1-T_{\text {ProbBroadcast_TX }}\right)-T_{\text {ProbBroadcast_Rx }}\right)-T_{\text {CSMA }}\right) / T_{\text {Interval }}\right)\right\rfloor \\
& * T_{\text {Sample }}
\end{aligned}
$$

Finally, the total time $T_{\text {ProbBroadcast_Idle, for which a }}$ node's radio remains in an idle state, is calculated to be:

$$
\begin{aligned}
& T_{\text {ProbBroadcast_Idle }} \\
= & \left(\left(\left(1-T_{\text {ProbBroadcast_Tx }}\right)-T_{\text {ProbBroadcast_Rx }}\right)-T_{\text {CSMA }}\right) \\
& -T_{\text {ProbBroadcast_Listen }}
\end{aligned}
$$

The above equations will be true only if the following condition is satisfied:

$$
T_{\text {ProbBroadcast_Listen }} \geq 0
$$

This is explained by the fact that a negative value for $T_{\text {ProbBroadcast_Listen }}$ implies that the bandwidth of the channel has been exceeded.

\subsection{Variable Preamble Length-Based Broadcasting Scheme}

The total power consumption of a node in the case of variable preamble length-based broadcasting scheme can be calculated from the durations for which the node's radio receiver or transmitter is turned on to perform the following functions: CSMA (4), transmission of the data packet (12), reception of a data packet (13) or (14), channel listening (15) and idling (16).

$$
\begin{aligned}
E_{\text {PreaBroadcast }}= & E_{\text {CSMA }}+E_{\text {PreaBroadcast_TX }}+E_{\text {PreaBroadcast_Rx }} \\
& +E_{\text {PreaBroadcast_Listen }}+E_{\text {PreaBroadcast_Idle }}
\end{aligned}
$$

where, $\left(E_{\text {CSMA }}=T_{\text {CSMA }} * P_{R x}\right),\left(E_{\text {PreaBroadcast_Tx }}=T_{\text {PreaBroad- }}\right.$ $\left.{ }_{\text {cast_TX }}{ }^{*} P_{T x}\right),\left(E_{\text {PreaBroadcast_Rx }}=T_{\text {PreaBroadcast_Rx }}{ }^{*} P_{R x}\right),\left(E_{\text {Prea- }}\right.$ Broadcast_Listen $\left.=T_{\text {PreaBroadcast_Listen }} * P_{R x}\right)$ and $\left(E_{\text {PreaBroadcast_Idle }}\right.$ $=T_{\text {PreaBroadcast_Idle }} * P_{\text {Idle }}$.

Before transmitting a data packet, a node will transmit a small preamble of fixed size followed by a data packet. This small preamble will always be less than the regular preamble sent in probability based broadcasting scheme as shown in Figure 1. The time which a node takes to transmit a packet can be represented by following equation;

$$
T_{\text {PreaBroadcast_TX }}=\left(T_{R x T x}+T_{S_{-} \text {Preamble }}+T_{T x}\right) * N_{\text {Pkts }}
$$

To receive a data packet, nodes will turn their receiver on if any channel activity is detected during periodic listening. These nodes will keep their receiver turned on until a packet is received and the total time, $T_{\text {PreaBroad- }}$ 
cast_Rx, for which the node receiver is turned on for the reception of a data packet is represented by following equation:

$$
T_{\text {PreaBroadcast_Rx }}=\left(\left(0.5 * T_{S_{-} \text {Preamble }}\right)+T_{R x}\right) * N_{\text {Pkts }} * N_{\text {Neigh }}
$$

In the worst case (when the receiver checks the channel just when the transmitting node starts sending the preamble) the total time, $T_{\text {PreaBroadcast_Rx }}$, for which the node receiver is turned on for the reception of a data packet is represented by following equation:

$$
T_{\text {PreaBroadcast_Rx }}=\left(\left(T_{S_{-} \text {Preamble }}\right)+T_{R x}\right) * N_{\text {Pkts }} * N_{\text {Neigh }}
$$

For the remainder of the time, when a node is neither receiving nor transmitting data, it performs periodic channel listening. The duration, $T_{\text {PreaBroadcast_Listen, for which }}$ the receiver is turned on for channel listening is calculated as follows:

$$
\begin{aligned}
& T_{\text {PreaBroadcast_Listen }} \\
= & \left\lfloor\left(\left(\left(\left(1-T_{\text {PreaBroadcast_TX }}\right)-T_{\text {PreaBroadcast_Rx }}\right)-T_{\text {CSMA }}\right) / T_{\text {Interval }}\right)\right\rfloor \\
& * T_{\text {Sample }}
\end{aligned}
$$

Finally, the total time, $T_{\text {PreaBroadcast_Idle, for which a }}$ node's radio remains in the idle state, is calculated to be:

$$
\begin{aligned}
& T_{\text {PreaBroadcast_Idle }} \\
& =\left(\left(\left(1-T_{\text {PreaBroadcast_Tx }}\right)-T_{\text {PreaBroadcast_Rx }}\right)-T_{\text {CSMA }}\right) \\
& \quad-T_{\text {PreaBroadcast_Listen }}
\end{aligned}
$$

As worst case assumptions are being used, the above equations will be true only if the following conditions are satisfied:

$$
\begin{gathered}
T_{\text {Interval }} \geq T_{S_{-} \text {Preamble }}+T_{I R x} \\
T_{\text {PreaBroadcast_Listen }} \geq 0
\end{gathered}
$$

The above mentioned conditions can be explained by the fact that a negative value for $T_{\text {PreaBroadcast_Listen }}$ implies that the bandwidth of the channel has been exceeded.

\section{Simulation Parameters Used and Results}

As the previously proposed variable preamble lengthbased broadcasting scheme [6] has been experimentally evaluated, we now present the preliminary results of the broadcasting schemes based on analytical study.

Table 2 shows the parameters used for the calculation of the energy consumption for the broadcasting schemes. These parameters are determined based on the CC2420 transceiver [8].

Packet broadcasting rate is 1 packet/sec. A data packet is of 32 bytes, time to transmit a byte is $0.000032 \mathrm{sec}$ and the data rate is $250 \mathrm{kbps}$. For the evaluation of probability-based broadcast the values of $T_{\text {Preamble }}$ are recalculated according to the $T_{\text {Interval }}(0.01-0.05 \mathrm{sec})$ based on formula (1).

Based on the parameters, the equations are solved to determine the energy consumption of the broadcasting nodes under different broadcasting conditions.

\subsection{Scenario 1}

Figures 2-4 show the energy consumption by the node's radios to communicate a packet between the nodes, while

Table 2. Parameters Used To Emulate CC2420.

\begin{tabular}{cc}
\hline Abbreviation & Unit \\
\hline$T_{R x T x}$ & $0.000192 \mathrm{Sec}$ \\
$T_{I R x}$ & $0.000192 \mathrm{Sec}$ \\
$T_{\text {RSSI }}$ & $0.000128 \mathrm{Sec}$ \\
$T_{\text {Sample }}$ & $0.00032 \mathrm{Sec}$ \\
$T_{\text {Guard }}$ & $0.00068 \mathrm{Sec}$ \\
$T_{c s m a}$ & $0.001 \mathrm{Sec}$ \\
$P_{R x}$ & $62.1 \mathrm{~mW}$ \\
$P_{T x}$ & $57.4 \mathrm{~mW}$ \\
$P_{I d l e}$ & $1.41 \mathrm{~mW}$ \\
\hline
\end{tabular}

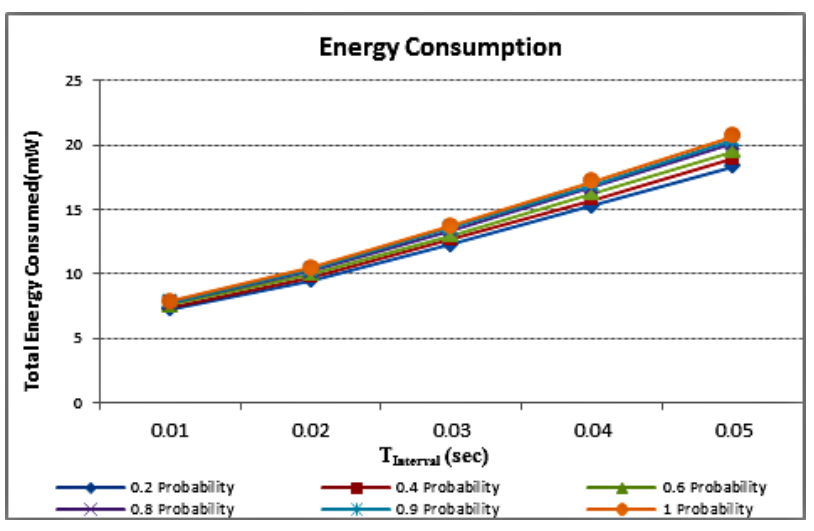

Figure 2. Energy Consumption (mW) Vs $T_{\text {Interval }}$ (sec).

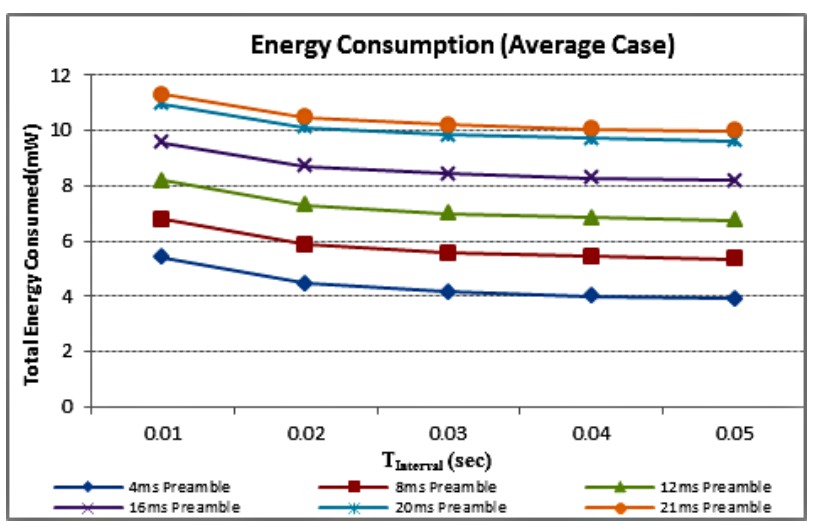

Figure 3. Energy Consumption (mW) Vs $T_{\text {Interval }}(\mathrm{sec})$. 


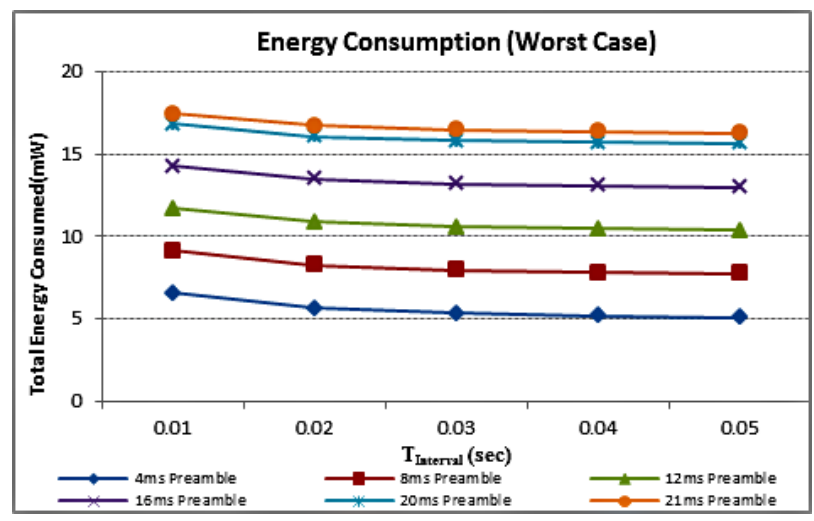

Figure 4. Energy Consumption (mW) Vs $T_{\text {Interval }}$ (sec).

the broadcasting node considers 10 nodes in the neighbourhood. The power consumptions by the radios is calculated for different sets of $T_{\text {Interval }}(0.01-0.05 \mathrm{sec}$ ).

Figure 2 shows the energy consumption (mW) versus $T_{\text {Interval }}$ (sec). It is observed that as the value of $T_{\text {Interval }}$ increases, the energy consumption increases linearly. This increase in the energy consumption is due to the long preamble used for increased $T_{\text {Interval }}$. Broadcasting a packet with a low probability shows less energy consumption as compared to broadcasting a packet with high probability. The difference in energy consumption of different probabilities of broadcasting is clearly visible at higher values of $T_{\text {Interval }}$.

Figures 3 and $\mathbf{4}$ show the energy consumption (mW) versus $T_{\text {Interval }}(\mathrm{sec})$ for average and worst cases respectively. The value of $T_{\text {Interval }}$ varies and the packets are broadcasted with different preamble length (4 ms to 21 ms). It is observed that as the value of $T_{\text {Interval }}$ increases the energy consumption decreases. Broadcasting a packet with a small preamble shows less power consumption compared to broadcasting a packet with a long preamble. A long preamble always results in high transmission and reception power consumption. The energy consumption of the radios in both cases decreases linearly as the $T_{\text {Inter- }}$ val increases. This energy saving in case of high $T_{\text {Interval }}$ is because of the small preambles used for broadcasting packets.

\subsection{Scenario 2}

Figures 5-7 show the energy consumption by the node's radios to communicate a packet between the nodes, while $T_{\text {Interval }}$ is considered as $0.02 \mathrm{sec}$. The power consumptions by the radios is calculated for a range of neighboring nodes (1 - 10 nodes).

Figure 5 shows the energy consumption (mW) versus number of neighbors. It is observed that as the number of neighbours of a broadcasting node increases, the energy consumption increases linearly. Broadcasting a packet with a low probability shows less energy consumption as

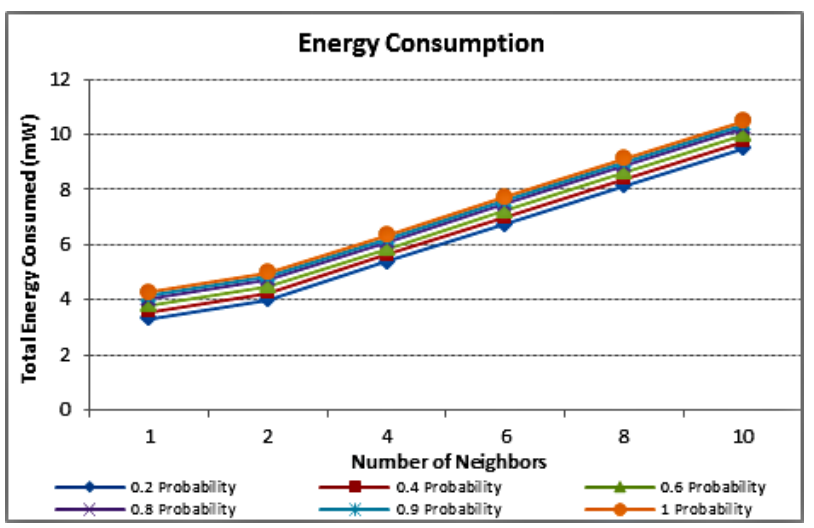

Figure 5. Energy Consumption (mW) Vs Number of Neighbors.

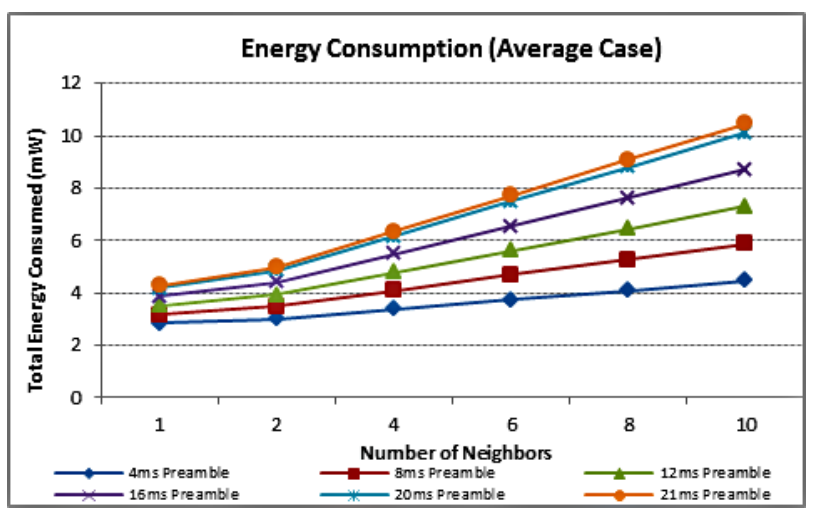

Figure 6. Energy Consumption (mW) Vs Number of Neighbors.

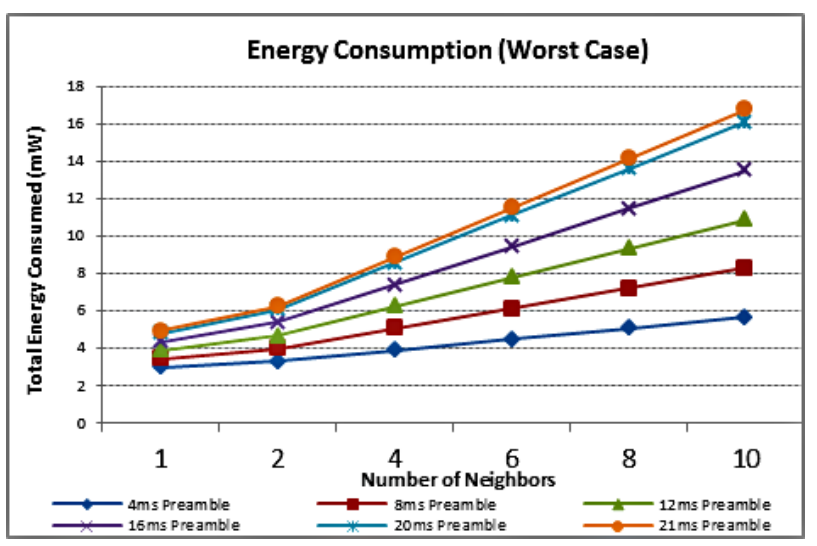

Figure 7. Energy Consumption (mW) Vs Number of Neighbors.

compared to broadcasting a packet with high probability.

Figures 6 and 7 show the energy consumption (mW) versus number of neighbors for average and worst cases respectively. The number of neighbors of a broadcasting node varies and the packets are broadcasted with different preamble length (4 ms to $21 \mathrm{~ms}$ ). It is observed that as the number of neighbors of a broadcasting node increases, the energy consumption increases linearly. Broad- 
casting a packet with a small preamble shows less power consumption compared to broadcasting a packet with a long preamble. A long preamble always results in high transmission and reception power consumption. The energy consumption of the radios in both cases increases as the neighboring nodes increases. But a higher energy consumption in worst case is due to the long preamble listening.

\section{Conclusion and Future Work}

In this paper, we have presented a comprehensive analytical model of the variable preamble length-based broadcasting scheme for wireless sensor networks. An analytical model for an existing probability-based broadcasting scheme is also presented. Analytical models do not consider the circuit and CPU power consumption in the presented equations. Our analytical study emphasizes the fact that a small preamble during broadcast can mitigate the need for higher energy consumption. Analytical results show that variable preamble length-based broadcasting scheme provides higher energy conservation in dense area networks, when compared to the probabilitybased scheme.

In future, we plan to collect these power consumption results by implementing these broadcasting algorithms on a physical test bed. Results obtained from analytical studies, simulations and physical test bed will be used to compare the broadcasting algorithms.

\section{REFERENCES}

[1] T. He, S. Krishnamurthy, L. Luo, T. Yan, L. Gu, R. Stoleru, G. Zhou, Q. Cao, P. Vicaire, J. A. Stankovic, T. F. Abdelzaher, J. Hui and B. Krogh, "VigilNet: An Inte- grated Sensor Network System for Energy-Efficient Surveillance,” ACM Transaction on Sensor Network, Vol. 2 2006, pp. 1-38. http://dx.doi.org/10.1145/1138127.1138128

[2] L. Selavo, A. Wood, Q. Cao, T. Sookoor, H. Liu, A. Srinivasan, Y. Wu, W. Kang, J. Stankovic, D. Young and J. Porter, "LUSTER: Wireless Sensor Network for Environmental Research," Proceedings of the 5th International Conference on Embedded Networked Sensor Systems, Sydney, 2007, pp. 103-116.

[3] N.Xu, S.Rangwala, K. K. Chintalapudi, D. Ganesan, A. Broad, R. Govindan and D. Estrin, "A Wireless Sensor Network for Structural Monitoring," Proceedings of the 2nd International Conference on Embedded Networked Sensor Systems, Baltimore, 2004, pp. 13-24.

[4] Y.-C. Tseng, S.-Y. Ni, Y.-S. Chen and J.-P. Sheu, "The Broadcast Storm Problem in a Mobile Ad-hoc Network," Wireless Network, Vol. 8, 2002, pp. 153-167. http://dx.doi.org/10.1023/A:1013763825347

[5] J. Polastre, J. Hill and D. Culler, "Versatile Low Power Media Access for Wireless Sensor Networks," Proceedings of the 2nd International Conference on Embedded Networked Sensor Systems, Baltimore, November 2004.

[6] A. Kumar and K. J. Wong, "A Variable Preamble Length-Based Broadcasting Scheme for Wireless Sensor Networks," 7th International Conference on Wireless Communications, Networking and Mobile Computing, Wuhan, 23-25 September 2011, pp. 1-4.

[7] K. J. Wong and D. K. Arvind, "SpeckMAC: Low-Power Decentralised MAC Protocols for Low Data rate Transmissions in Specknets," Proceedings of the 2nd International Workshop on Multi-Hop Ad Hoc Networks: From Theory to Reality, Florence, 2006, pp. 71-78.

[8] Texas Instrument, 2.4 GHz IEEE 802.15.4/ZigBee-ready RF Transceiver. http://focus.ti.com/lit/ds/symlink/cc2420.pdf. 